\title{
Throughput-Oriented Full-Duplex Cognitive Radio Network Parameter Optimization
}

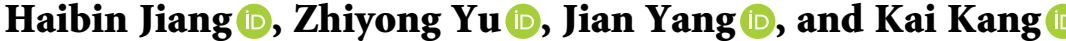

Xi'an Research Institute of High-Tech, Xi'an 710025, China

Correspondence should be addressed to Haibin Jiang; haibin_jiang@foxmail.com

Received 25 June 2021; Revised 12 October 2021; Accepted 25 November 2021; Published 4 January 2022

Academic Editor: Giuseppina Monti

Copyright (C) 2022 Haibin Jiang et al. This is an open access article distributed under the Creative Commons Attribution License, which permits unrestricted use, distribution, and reproduction in any medium, provided the original work is properly cited.

Full-duplex cooperative spectrum sensing (FD-CSS) is an important research field in the field of spectrum sensing. In the FD-CSS network, the secondary user (SU) senses the usage status of the authorized spectrum by the primary user (PU) through the sensing channel and then reports the perceived data to the fusion center (FC) through the reporting channel. The FC makes a comprehensive judgment after summarizing the data through the fusion algorithm. In the secondary network with SU, throughput is an important index to measure the performance of the network. Taking throughput as the optimization goal, this paper theoretically deduces and verifies the optimal data fusion algorithm in cooperative spectrum sensing (CSS), the threshold of optimal energy detection, and the optimal transmission power of SU in the secondary network. The simulation results show the correctness of the results in this paper.

\section{Introduction}

In order to solve the problem of spectrum shortage, people combine cognitive radio (CR) technology with in-band fullduplex (IBFD) technology and propose full-duplex cognitive radio (FDCR) technology. Multiuser CSS can use spatial diversity to improve spectrum sensing performance. Therefore, FD-CSS is an important research direction in the future. In half-duplex cognitive radio (HDCR) networks, secondary user (SU) senses the authorized channel of primary user (PU) and accesses the channel opportunistically [1]. At this time, SU divides the continuous timeslot into two sections; the first section is used for spectrum sensing, and the second half is used for data transmission, which is generally called "listen before speak (LBT)" strategies. The strategies limit the spectrum efficiency of CR networks and increase the risk of data conflict [2].

In-band full-duplex (IBFD) allows communication terminals to transmit and receive data simultaneously in the same frequency band, which can theoretically double the utilization of the frequency band. It was a key technology of $5 \mathrm{G}$ together with cognitive radio (CR), massive multiple input multiple output (mMIMO), and other technologies.
Paper [3] presents a smart composition utilizing both mMIMO and in-band full-duplex (IBFD), called IBFD mMIMO, which substantially enhances the spectral efficiency (SE). Paper [4] studies the design of hybrid time switching and power splitting simultaneous wireless information and power transfer (SWIPT) protocol for IBFD mMIMO. Paper [5] studies the capacity improvement of full-duplex (FD) device-to-device (D2D) under cellular networks. Under sufficient self-interference cancellation (SIC), the capacity improvement of FD-D2D communication is much greater than that of traditional HD-D2D communication. Paper [6] proposes applying IBFD to CR to improve the throughput of SU. It is verified that the throughput of SU is higher than HDCR.

The main difficulty of realizing IBFD lies in the suppression of self-interference, because in the same channel the transmitter will produce greater self-interference to the receiver when it outputs. Recent studies [7-13] have proved the feasibility of short-range full-duplex wireless communication. By combining passive suppression and active cancellation, self-interference can be reduced to an acceptable level, and the remaining interference is called residual self-interference (RSI). 
In FDCR, SU can sense spectrum and transmit data over a given channel at the same time, commonly referred to as the "Listening and Speaking (LAT)" strategies. Compared with HDCR, FDCR improves network secondary throughput and reduces data conflict probability [14]. This paper presents FD-CSS due to the instability of local detection performance, in which the local spectrum sensing results of SUs are uploaded to the FC, which makes a decision on the presence of PU through the FC [15]. At present, the research on FD-CRN focuses on how to improve secondary throughput. Document [16] studies FDCR in noncooperative mode. By comparing with noncooperative HDCR, it is found that FDCR has higher secondary throughput than HDCR at the same transmission power. Paper [17] researches the performance of LAT strategies under collaboration awareness and compares it with traditional LBT strategies. Paper [18] improves throughput in cooperation and noncooperation scenarios by optimizing detection thresholds. Document [19] discusses throughput maximization under energyconstrained conditions.

In this paper, we studied the effects of parameters in cognitive radio networks on throughput. The main contributions of this paper are as follows:

(i) In the actual cognitive radio network, the detection probability of SUs spectrum sensing has a lower limit, so the range of detection threshold changes is limited. We prove that the range of detection threshold is also limited. In order to obtain the secondary network throughput, this paper studies how to obtain it by setting the detection of spectrum sensing. By comparison, this method improves the performance of cognitive radio network.

(ii) In FD-CSS, the sensing node sends the sensing results to the $\mathrm{FC}$, which gathers the result through the $n$ - out - of - $N$ rule and feeds back the result to each node. This paper studies how to set $n$ in the $n$ - out - of $-N$ rule to obtain the optimal sensing performance.

(iii) For FD-CSS, when the transmission power is low, the secondary network throughput is better than HD-CSS. But, due to the existence of RSI, there are pole in the transmission power-throughput curve when the transmit power increases continuously. From the point of view of energy efficiency, this pole has the highest energy efficiency, which is called local optimal transmit power. In this paper, we use Newton method to obtain it.

The remainder of the paper is organized as follows. Section 2 describes the system model and the concept of FDCSS. In Section 3, we discuss the relationship between important parameters and optimal secondary throughput. In Section 4, simulation results are presented to verify the analytical results and conclusions are given in Section 5.

\section{System Model}

As shown in Figure 1, we consider a network with one PU, one FC, and $K$ SUs under a FD-CSS scheme. In this scheme, PU has priority to use an authorized spectrum. FC is used to receive the sensing results sent by the local sensing node and control and coordinate the whole cooperation process. SUs are equipped with two antennas and have self-interference cancellation (SIC) capability. Ant ${ }_{1}$ is in charge of spectrum sensing and $\mathrm{Ant}_{2}$ is in charge of data transmission. SU periodically senses the local spectrum for a specific channel and makes a local decision about the presence of the PU based on its observation. We assume that SUs sample the specific spectrum with frequency $f_{s}$ in time slot $T$; the number of sampling points is $N_{s}=f_{s} T$. The local decisions are to be sent to the FC in different time slots based on a Time Division Multiple Access (TDMA) scheme. FC uses the preset fusion rule to make the final decision on the PU state. When the decision result is that the authorized channel is idle, a SU is arranged to transmit data. We assume that the $\mathrm{SU}$ is $\mathrm{SU}_{1}$. When the decision result is not idle, all SUs continue to maintain the sensing state.

Local sensing-transmission of $\mathrm{SU}_{1}$ can be expressed as a hypothesis testing problem. When the authorized channel is not occupied by PU, we assume that $H_{00}$ and $H_{01}$ represent when $\mathrm{SU}_{1}$ transmits and when $\mathrm{SU}_{1}$ does not transmit, respectively. When the authorized channel is occupied by PU, we assume that $H_{10}$ and $H_{11}$ represent when $\mathrm{SU}_{1}$ transmits and when $\mathrm{SU}_{1}$ does not transmit, respectively.

The signal $y_{i}(n)$ sampled by $\mathrm{SU}_{i}$ at time nth can be written as

$$
y_{i}(n)= \begin{cases}u_{i}(n), & H_{00}, \\ x_{i}(n)+u_{i}(n), & H_{01}, \\ h_{1, i} s_{i}(n)+u_{i}(n), & H_{10} \\ x_{i}(n)+h_{1, i} s_{i}(n)+u_{i}(n), & H_{11}\end{cases}
$$

where $u_{i}(n)$ is the additive white Gaussian noise (AWGN) at the sensing channel, $x_{i}(n)$ is the sampling value of PU transmitted signal received by the sensing node at the $n$th time, $s_{i}(n)$ is the signal of $\mathrm{SU}_{1}$ in data transmission, and $h_{1, i}$ denotes the channel fading amplitude between $\mathrm{SU}_{i}$ and $\mathrm{SU}_{1}$. This paper mainly assumes the following:

(a) The noise $u_{i}(n)$, primary signal $x_{i}(n)$, and $\mathrm{SU}_{1}$ transmit signal $s(n)$ is Circularly Symmetric Complex Gaussian (CSCG) variable random process with mean zero and variance $E\left[\left|u_{i}(n)\right|^{2}\right]=\delta_{u}^{2}$, $E\left[\left|x_{i}(n)\right|^{2}\right]=\delta_{p}^{2}$ and $E\left[\left|s_{i}(n)\right|^{2}\right]=\delta_{s}^{2}$.

(b) It is assumed that the channel $h_{1, i}$ is Rayleigh faded with zero mean and variance $\chi_{1, i}^{2} \delta_{s}^{2}, \chi_{1, i}$ reflects the degree of SIC. In this paper, we assume that $\chi_{1, i}^{2}=\chi^{2}$.

(c) We denote $\gamma_{p}=\left(\delta_{p}^{2} / \delta_{u}^{2}\right)$ as the received signal-tonoise ratio (SNR) of $\mathrm{SU}_{i}$. We denote $\gamma_{s}=\left(\chi^{2} \delta_{s}^{2} / \delta_{u}^{2}\right)$ as the received interference-to-noise ratio (INR) of $\mathrm{SU}_{i}$. 


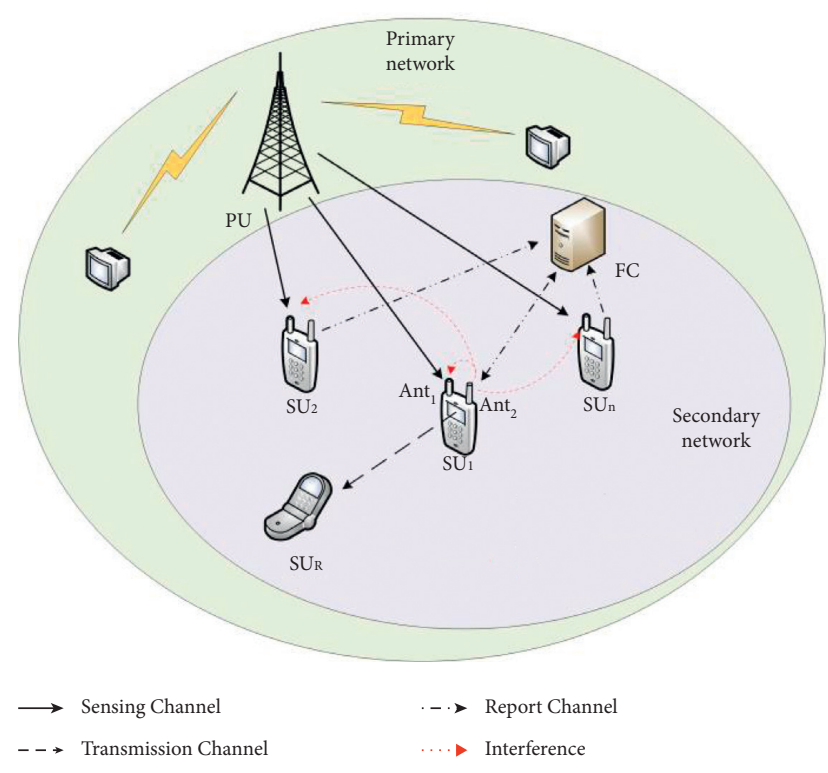

FIGURE 1: Full-duplex cognitive radio with cooperative spectrum sensing.

2.1. Energy Detection. Due to the fact that energy detection does not require prior information of the PU signal and is easy to implement, SU uses energy detection for local spectrum sensing. Then the test statistic $Y_{i}$ is

$$
Y_{i}=\frac{1}{N_{s}} \sum_{n=1}^{N_{s}}\left|y_{i}(n)\right|^{2}
$$

When we select the threshold as $\eta$, if $Y_{i}>\eta$, the judgment channel is occupied by the PU. And if $Y_{i} \geq \eta$, the judgment channel is not occupied by the PU. Consider that the perceived signal is affected by $\mathrm{SU}_{1}$ transmission data. $\eta_{1}$ and $\eta_{0}$, respectively, represent the sensing threshold of $\mathrm{SU}_{i}$ when $\mathrm{SU}$ is active and not.

Under $H_{00}$ hypothesis, energy detection can be expressed as

$$
Y_{i} \stackrel{H_{10}}{\gtrless} \eta_{00} .
$$

When the sample number $N_{s}$ is large enough, according to central limit theorem (CLT), the probability density function (PDF) of $Y_{i}$ can be approximated to a Gaussian distribution. Therefore,

$$
\begin{aligned}
E\left[Y_{i}\right] & =\mathrm{E}\left|u_{i}(n)\right|^{2}=\delta_{u}^{2}, \\
\operatorname{var}\left[Y_{i}\right] & =\frac{1}{N_{s}}\left[E\left|u_{i}(n)\right|^{4}-\delta_{u}^{4}\right] .
\end{aligned}
$$

Due to $u_{i}(n) \quad$ is CSCG, $E\left|u_{i}(n)\right|^{4}=2 \delta_{u}^{4} \quad$ and $\operatorname{var}\left[Y_{i}\right]=\left(1 / N_{s}\right) \delta_{u}^{4}$. The distribution of random variable $Y_{i}$ can be expressed as

$$
Y_{i} \sim \begin{cases}N\left(\sigma_{u}^{2}, \frac{\delta_{u}^{4}}{N_{s}}\right), & H_{00}, \\ N\left(\left(1+\gamma_{p, i}\right) \delta_{u}^{2}, \frac{\left(1+\gamma_{p, i}\right)^{2} \delta_{u}^{4}}{N_{s}}\right), & H_{10},\end{cases}
$$

where $\gamma_{p, i}$ means signal-to-noise ratio (SNR) of PU under various assumptions. Similarly, the mean and variance are shown in Table 1.

The false alarm probability and detection probability can be expressed as

$$
\begin{aligned}
& P_{f, i}^{0}=\operatorname{Pr}\left(Y_{0, i}>\eta_{0, i} \mid H_{00}\right), \\
& P_{d, i}^{0}=\operatorname{Pr}\left(Y_{0, i}>\eta_{1, i} \mid H_{10}\right),
\end{aligned}
$$

according to equations (4) and (5). The false alarm probability can be expressed as

$$
\begin{aligned}
P_{f, i}^{0} & =\operatorname{Pr}\left(\frac{Y_{0, i}-\delta_{u}^{2}}{\sqrt{\delta_{u}^{4} / N_{s}}}>\frac{\eta_{0, i}-\delta_{u}^{2}}{\sqrt{\delta_{u}^{4} / N_{s}}} \mid H_{00}\right) \\
& =Q\left(\left(\frac{\eta_{0, i}}{\delta_{u}^{2}}-1\right) \sqrt{N_{s}}\right),
\end{aligned}
$$

where $Q(\cdot)=(1 / \sqrt{2 \pi}) \int_{x}^{\infty} \exp \left(-t^{2} / 2\right) \mathrm{d} t$. Similarly, it can be obtained that

$$
P_{d, i}^{0}=Q\left(\left(\frac{\eta_{0, i}}{\left(1+\gamma_{p, i}\right) \delta_{u}^{2}}-1\right) \sqrt{N_{s}}\right) .
$$

In practical application, it is necessary to ensure that the SU detection probability is not less than a certain value. For example, the 802.22 wireless regional area network (WRAN) standard requires a detection probability of at least $90 \%$ for TV signals [20]. For target detection probability $\bar{P}_{d}$, the energy detection threshold and false alarm probability can be expressed as

$$
\begin{aligned}
\eta_{0, i}\left(\bar{P}_{d}\right) & =\left(\frac{Q^{-1}\left(\bar{P}_{d}\right)}{\sqrt{N_{s}}}+1\right)\left(1+\gamma_{p, i}\right) \delta_{u}^{2}, \\
P_{f, i}^{0}\left(\bar{P}_{d}\right) & =Q\left(Q^{-1}\left(\bar{P}_{d}\right)\left(1+\gamma_{p, i}\right)+\gamma_{p, i} \sqrt{N_{s}}\right) .
\end{aligned}
$$

With the properties in Table 1, the local probabilities of miss detection and false alarm when $\mathrm{SU}_{1}$ transmits can be expressed as

$$
\begin{aligned}
& \eta_{1, i}\left(\bar{P}_{d}\right)=\left(\frac{Q^{-1}\left(\bar{P}_{d}\right)}{\sqrt{N_{s}}}+1\right)\left(1+\gamma_{p, i}+\gamma_{s, i}\right) \delta_{u}^{2}, \\
& P_{f, i}^{1}\left(\bar{P}_{d}\right)=Q\left(Q^{-1}\left(\bar{P}_{d}\right)\left(1+\frac{\gamma_{p, i}}{1+\gamma_{s, i}}\right)+\frac{\gamma_{p, i}}{1+\gamma_{s, i}} \sqrt{N_{s}}\right) .
\end{aligned}
$$


TABLE 1: Statistical properties of $Y_{i}$.

\begin{tabular}{lcc}
\hline Hypothesis & $E\left[Y_{i}\right]$ & $\operatorname{var}\left[Y_{i}\right]$ \\
\hline$H_{00}$ & $\delta_{u}^{2}$ & $\delta_{u}^{4} / N_{s}$ \\
$H_{01}$ & $\left(1+\gamma_{p}\right) \delta_{u}^{2}$ & $\left(1+\gamma_{p}\right)^{2} \delta_{u}^{4} / N_{s}$ \\
$H_{10}$ & $\left(1+\gamma_{s}\right) \delta_{u}^{2}$ & $\left(1+\gamma_{s}\right)^{2} \delta_{u}^{4} / N_{s}$ \\
$H_{11}$ & $\left(1+\gamma_{s}+\gamma_{p}\right) \delta_{u}^{2}$ & $\left(1+\gamma_{s}+\gamma_{p}\right)^{2} \delta_{u}^{4} / N_{s}$ \\
\hline
\end{tabular}

2.2. Decision Fusion. In FD-CSS, cooperative user $\mathrm{SU}_{i}$ participating in local sensing sends their sensing results to FC, and FC uses preset data decision fusion rule to determine whether PU exists or does not exist. Due to the fact that hard data fusion is simple and easy to implement, this paper uses hard data fusion rule. In hard data fusion, 1-bit decision result $\xi_{i}\left(\xi_{i}=1\right.$ means $\mathrm{PU}$ exists; $\xi_{i}=0$ means $\mathrm{PU}$ does not exist) is sent to FC. FC adopts the global decision rule of $n$ - out - of $-N$ to express the following binary decision, indicating that PU exists in $n$ of $N$ SUs; then FC determines that PU exists. The $n-$ out - of $-N$ rule can be expressed as

$$
\sum_{i=1}^{N} \xi_{i} \gtrless_{H_{0}}^{H_{1}} n,
$$

where $H_{1}$ denotes that PU exists and $H_{0}$ denotes that PU does not exist in FC judgment. When $n=1$, the fusion rule is OR rule; when $n=N$, the fusion rule is AND rule.

2.3. Throughput. As shown in Figure 1, there are two cases of spectrum waste. First, when the PU is not active in the authorized channel, the $\mathrm{SU}_{1}$ sensing antenna $\mathrm{Ant}_{1}$ has a detection error. Detect the status error of $\mathrm{PU}$ as active second; PU exits from the authorized channel when $\mathrm{SU}_{1}$ sensing spectrum. This paper considers the situation when the PU state changes slowly. At this time, the second case can be ignored. We assume that different SUs have the same threshold at the same time:

$$
\begin{aligned}
& \eta_{0,1}=\eta_{0,2}=\cdots=\eta_{0, K}=\eta_{0} \\
& \eta_{1,1}=\eta_{1,2}=\cdots=\eta_{1, K}=\eta_{1},
\end{aligned}
$$

combining with equations (9)-(12),

$$
\begin{aligned}
& P_{f, 1}^{s}=P_{f, 2}^{s}=\cdots=P_{f, K}^{s}=P_{f}^{s}, \\
& P_{d, 1}^{s}=P_{d, 2}^{s}=\cdots=P_{d, K}^{s}=P_{d}^{s},
\end{aligned}
$$

where $s=0,1$.

In [14], Liao derived the false alarm probability and detection probability of a single full-duplex cognitive user:

$$
\begin{aligned}
& P_{f}=\frac{P_{f}^{1}}{1-P_{f}^{0}+P_{f}^{1}}, \\
& P_{d}=\frac{P_{d}^{1}}{1-P_{d}^{0}+P_{d}^{1}},
\end{aligned}
$$

combining with equation (14), the false alarm probability and detection probability in FD-CSS are

$$
\begin{aligned}
& \mathbb{P}_{f}=\sum_{j=n}^{K}\left(\begin{array}{c}
K \\
j
\end{array}\right) P_{f}^{j}\left(1-P_{f}\right)^{K-j}, \\
& \mathbb{P}_{d}=\sum_{j=n}^{K}\left(\begin{array}{c}
K \\
j
\end{array}\right) P_{d}^{j}\left(1-P_{d}\right)^{K-j} .
\end{aligned}
$$

The spectrum efficiency of cognitive radio network is an important index to reflect its performance. According to the false alarm probability of FD-CSS and Shannon's formula, the throughput is defined:

$$
\Re=\left(1-\mathbb{P}_{f}\right) \log _{2}\left(1+\gamma_{t}\right),
$$

where $\gamma_{t}=\left(\delta_{s}^{2} \delta_{t}^{2} / \delta_{u}^{2}\right)$ denotes the transmission SNR of $\mathrm{SU}_{1}$ and $\delta_{t}^{2}$ denotes Rayleigh channel variance from $\mathrm{SU}_{1}$ to receiver.

\section{Parameter Optimization Analysis}

It can be concluded from the above that the throughput of cognitive radio network depends on the power of the transmitted signal $\delta_{t}^{2}$, the thresholds $\eta_{1}$ and $\eta_{2}$, and the value $n$ in the $n$-out - of $-N$ fusion rule. This paper mainly studies how to maximize the throughput through optimization of these three parameters.

3.1. Threshold-Throughput. In order to protect the main network from the interference of cognitive network, the detection probability of cognitive network needs to be properly constrained. In practical application, the constraint condition required by the main network operation protection is usually close to but less than 1. For example, in 802.22 wireless regional area network (WRAN), a detection probability of at least $90 \%$ for TV signals is required. In this paper, the detection probability of the limiting system should not be less than $\widehat{P}_{d}$. The problem formulation of the sensing-throughput trade-off with FD-CSS is given as

$$
\begin{gathered}
\max \quad \Re\left(n, \eta_{0}, \eta_{1}, \delta_{s}^{2}\right), \\
\text { s.t. } P_{d}^{0} \geq \widehat{P}_{d}, P_{d}^{1} \geq \widehat{P}_{d}, \\
n=1,2, \ldots, K .
\end{gathered}
$$

When the constraint (23) is equal, the optimal solution is obtained. The proof is as follows.

Proof. Hypothesis $P_{d}^{1}=P_{d}^{0}=\widehat{P}_{d}$, we denote here $\eta_{0}=\widehat{\eta}_{0}$, $\eta_{1}=\widehat{\eta}_{1}$.

(1) When $P_{d}^{0} \geq \widehat{P}_{d}$ and $P_{d}^{1}=\widehat{P}_{d}$, we get $\eta_{0} \leq \widehat{\eta}_{0}$ and $\eta_{1}=$ $\widehat{\eta}_{1}$ from equations (10) and (12).

Due to $P_{f}^{0}\left(\eta_{0}\right)=Q\left(\left(\left(\eta_{0} / \delta_{u}^{2}\right)-1\right) \sqrt{N_{s}}\right)$ and $Q(\cdot)$ is a decreasing function,

Then $P_{f}^{0}\left(\eta_{0}\right) \geq P_{f}^{0}\left(\widehat{\eta}_{0}\right)$, Joint $P_{f}=P_{f}^{1} /\left(1-P_{f}^{0}+P_{f}^{1}\right)$, we get 


$$
P_{f}\left(\eta_{0}\right) \geq P_{f}\left(\widehat{\eta}_{0}\right) .
$$

(2) When $P_{d}^{0}=\widehat{P}_{d}$ and $P_{d}^{1} \geq \widehat{P}_{d}$, we get $\eta_{0}=\widehat{\eta}_{0}$ and $\eta_{1} \leq \widehat{\eta}_{1}$ from equations (10) and (12).

So $P_{f}^{1}\left(\eta_{0}\right) \geq P_{f}^{1}\left(\widehat{\eta}_{0}\right)$, Joint $P_{f}=P_{f}^{1} /\left(1-P_{f}^{0}+P_{f}^{1}\right)$, we get equation (25).

We take the partial derivative of equation (19):

$\frac{\partial \mathbb{P}_{f}}{\partial P_{f}}=n P_{f}^{n-1}\left(1-P_{f}\right)^{K-n}-(K-n) P_{f}^{n}\left(1-P_{f}\right)^{K-n-1}$.

Generally speaking, $P_{f}$ is very small, so $1-P_{f} \longrightarrow 1$; equation (19) can be expressed as

$$
\frac{\partial \mathbb{P}_{f}}{\partial P_{f}}=\left[n-P_{f}(K-n)\right] P_{f}^{n-1}>0 .
$$

Joining equations (21), (25), and (27), we obtain

$$
\begin{gathered}
\mathbb{P}_{f} \geq \widehat{\mathbb{P}}_{f}, \\
\mathfrak{R}\left(n, \eta_{0}, \eta_{1}, \delta_{s}^{2}\right) \leq \widehat{\mathfrak{R}}\left(n, \widehat{\eta}_{0}, \widehat{\eta}_{1}, \delta_{s}^{2}\right) .
\end{gathered}
$$

Equation (29) is reduced to

$$
\begin{gathered}
\max \Re\left(n, \eta_{0}, \eta_{1}, \delta_{s}^{2}\right), \\
\text { s.t. } P_{d}^{0}=\widehat{P}_{d}, P_{d}^{1}=\widehat{P}_{d}, \\
n=1,2, \ldots, K .
\end{gathered}
$$
that

Applying this to equations (10) and (12), we can derive

$$
\begin{gathered}
\eta_{0}\left(\widehat{P}_{d}\right)=\left(\frac{Q^{-1}\left(\widehat{P}_{d}\right)}{\sqrt{N_{s}}}+1\right)\left(1+\gamma_{p}\right) \delta_{u}^{2}, \\
\eta_{1}\left(\widehat{P}_{d}\right)=\left(\frac{Q^{-1}\left(\widehat{P}_{d}\right)}{\sqrt{N_{s}}}+1\right)\left(1+\gamma_{p}+\gamma_{s}\right) \delta_{u}^{2} .
\end{gathered}
$$

When $\mathrm{SU}_{1}$ transmits data, the detection threshold increases due to self-interference, so the false alarm probabilities $P_{f}^{0}$ and $P_{f}^{1}$ are different:

$$
\begin{aligned}
& P_{f}^{0}\left(\widehat{P}_{d}\right)=Q\left(Q^{-1}\left(\widehat{P}_{d}\right)\left(1+\gamma_{p}\right)+\gamma_{p} \sqrt{N_{s}}\right) \\
& P_{f}^{1}\left(\widehat{P}_{d}\right)=Q\left(Q^{-1}\left(\widehat{P}_{d}\right)\left(1+\frac{\gamma_{p}}{1+\gamma_{s}}\right)+\frac{\gamma_{p}}{1+\gamma_{s}} \sqrt{N_{s}}\right) .
\end{aligned}
$$

3.2. Decision Fusion-Throughput. In this section, we mainly discuss the relationship between the value of $n$ and the throughput in the $n$-out - of $-N$ fusion rule. Applying (30) to equation (31), we obtain

$$
\begin{aligned}
& \max \Re\left(n, \delta_{s}^{2}\right), \\
& \text { s.t. } n=1,2, \ldots, K,
\end{aligned}
$$

where

$$
\begin{aligned}
\widehat{\mathfrak{R}}\left(n, \delta_{s}^{2}\right) & =\left(1-\mathbb{P}_{f}\right) \log _{2}\left(1+\frac{\delta_{s}^{2} \delta_{t}^{2}}{\delta_{u}^{2}}\right) \\
& =\left[1-\sum_{j=n}^{K}\left(\begin{array}{c}
K \\
j
\end{array}\right) P_{f}^{j}\left(1-P_{f}\right)^{K-j}\right] \log _{2}\left(1+\frac{\delta_{s}^{2} \delta_{t}^{2}}{\delta_{u}^{2}}\right) .
\end{aligned}
$$

From (35), let $\delta_{s}^{2}$ remain unchanged.

(1) When $\gamma_{P}$ (or SNR) $\longrightarrow+\infty$, from equations (35) and (36) we obtain $P_{f}^{1} \longrightarrow 0, P_{f}^{0} \longrightarrow 0$. Applying this to equations (19) and (21), we obtain

$$
\widehat{\Re}\left(n, \delta_{s}^{2}\right) \longrightarrow \log _{2}\left(1+\frac{\delta_{s}^{2} \delta_{t}^{2}}{\delta_{u}^{2}}\right) .
$$

In fact, it is not necessary for $\gamma_{P} \longrightarrow+\infty$, when $\gamma_{P} \geq \widehat{\gamma}_{p}$; in this case, no matter what the value of $n$ is, $\mathfrak{R}\left(n, \delta_{s}^{2}\right) \equiv \log _{2}\left(1+\left(\delta_{s}^{2} \delta_{t}^{2} / \delta_{u}^{2}\right)\right)$ is satisfied. In this case, the throughput is independent of $n$.

(2) When $0<\gamma_{P} \leq \widehat{\gamma}_{p}$, due to $0<P_{f}<1$, so

$$
\frac{\partial \widehat{\Re}\left(n, \delta_{s}^{2}\right)}{\partial n}=\left(\begin{array}{l}
K \\
n
\end{array}\right) P_{f}^{n}\left(1-P_{f}\right)^{K-n}>0 .
$$

When (41) is satisfied, $\widehat{\mathfrak{R}}\left(n, \delta_{s}^{2}\right)$ increases with the increase of $n$. In this case, when $n=K, \widehat{\mathfrak{R}}\left(n, \delta_{s}^{2}\right)$ reaches the maximum.

In conclusion, when $0<\gamma_{P} \leq \widehat{\gamma}_{p}, \widehat{\Re}\left(n, \delta_{s}^{2}\right)$ increases with the increase of $n$, when $\gamma_{P} \geq \hat{\gamma}_{p}$, no matter what the value of $n$ is, $\widehat{\Re}\left(n, \delta_{s}^{2}\right) \equiv \log _{2}\left(1+\left(\delta_{s}^{2} \delta_{t}^{2} / \delta_{u}^{2}\right)\right)$. Therefore, when $n=K$, $\widehat{\mathfrak{R}}\left(n, \delta_{s}^{2}\right)$ can always reach the maximum.

3.3. Transmit Power-Throughput. It can be seen from the previous section that when the fusion criterion adopts AND rule, $\widehat{\mathfrak{R}}\left(n, \delta_{s}^{2}\right)$ achieves the optimal throughput.

Equation (37) is reduced to

$$
\begin{aligned}
\left.\widetilde{\mathbb{P}}_{f}\right|_{n=K} & =P_{f}^{K}, \\
\left.\widetilde{\mathfrak{R}}\left(\delta_{s}^{2}\right)\right|_{n=K} & =\left(1-P_{f}^{K}\right) \log _{2}\left(1+\frac{\delta_{s}^{2} \delta_{t}^{2}}{\delta_{u}^{2}}\right) .
\end{aligned}
$$

According to equation (17), we can get when $\delta_{s}^{2}$ increases, $\left(1-P_{f}^{K}\right)$ decreases and $\log _{2}\left(1+\left(\delta_{s}^{2} \delta_{t}^{2} / \delta_{u}^{2}\right)\right)$ increases. From paper [13], when $\mathrm{SU}_{1}$ transmitting power $\delta_{t}^{2}$ satisfies the following equation:

$$
\exp \left(-\frac{\rho^{2}}{2}\right) \frac{\left(\gamma_{t}+1\right) \ln \left(\gamma_{t}+1\right)}{\left(\gamma_{s}+1\right)^{2}}<\frac{\sqrt{2 \pi} \delta_{t}^{2}}{\Xi}\left(1-P_{f}^{0}+Q(\rho)\right),
$$

where 


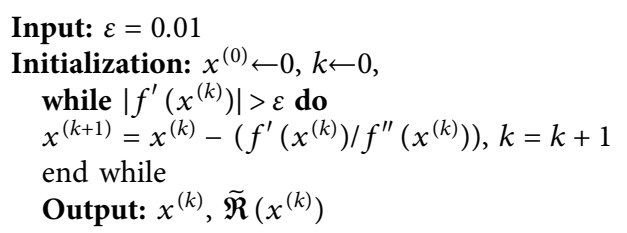

Algorithm 1: Find the optimal $x=\delta_{s}^{2}$ that makes $f(x)=1 / \widetilde{\Re}$ reach the extreme point.

$$
\begin{aligned}
\rho & =-Q^{-1}\left(P_{m}\right)\left(\frac{\gamma_{p}}{\gamma_{s}+1}+1\right)+\frac{\gamma_{s}}{\gamma_{s}+1} \sqrt{N_{s}}, \\
Q(\rho) & =P_{f}^{1}, \\
\Xi & =\gamma_{p} \chi^{2}\left(Q^{-1}\left(1-P_{m}\right)+\sqrt{N_{s}}\right),
\end{aligned}
$$

there is a local optimal transmit power for $\widetilde{\mathfrak{R}}\left(\delta_{s}^{2}\right)$. An example of using Newton method to find the local optimal $\delta_{s}^{2}$ is given in Algorithm 1, .

\section{Computer Simulations}

In this section, we use Monte Carlo simulation to checking the previous conclusions. We set the slot duration $T=10 \mathrm{~ms}$, the sampling frequency $f_{s}=6 \mathrm{MHz}$, the noise variance $\delta_{u}^{2}=-10 \mathrm{~dB}$, and the number of perceptive users $K=10$ as parameters. Local detection uses energy detection, and the number of Monte Carlo trials used to calculate the probability is 10000 .

Figure 2 shows the variation of FD-CSS false alarm probability with relative threshold when $\gamma_{p}=0 \mathrm{~dB}, 1 \mathrm{~dB}$, $2 \mathrm{~dB}$ where the SIS factor $\chi^{2}=0.1$, the minimum detection probability $\widehat{P}_{d}=0.9999$, and the relative transmission power $\delta_{s}^{2} / \delta_{u}^{2}=10 \mathrm{~dB}$. We set the relative threshold $\widehat{\eta}_{0}=0.4099$, $\widehat{\eta}_{1}=10.2581$ when the minimum detection probability $\widehat{P}_{d}=$ 99.99\% from equations (33) and (34). As shown in Figure 2, when the detection threshold decreases, the false alarm probability increases as (25). The main reason is that when the detection threshold is lower, the FD-CSS is easier to judge the received signal as the authorized channel. It can also be seen from Figure 2 that when the $\gamma_{p}$ signal-to-noise ratio increases, the false alarm probability increases, because, in the received signals, the authorized channel has more energy and is easier to be detected.

Figure 3 shows the throughput $\mathfrak{R}$ in the FD-CSS scenario versus the sensing SNR of the PU signal with the constraint on minimum detection probability $\widehat{P}_{d}=99.99 \%$ and the relative transmission power $\left(\gamma_{s}\right)=10 \mathrm{~dB}$, for the detection threshold $\eta_{1}=\widehat{\eta}_{1}, \widehat{\eta}_{1}-0.05, \widehat{\eta}_{1}-0.1$. For high SNR (in this scenario, less than $5 \mathrm{~dB}$ ), the throughput is approximately the upper bound. Under conditions of low SNR, the throughput decreases as the detection threshold decreases. For example, when SNR is $2 \mathrm{~dB}$ and $\eta_{1}=\widehat{\eta}_{1}$, the throughput is $\widehat{\Re}=5.4 \mathrm{bps} / \mathrm{Hz}$, whereas when $\eta_{1}=\widehat{\eta}_{1}-0.05$, the throughput is $\widehat{\Re}=3.1 \mathrm{bps} / \mathrm{Hz}$.

We also compare full-duplex noncooperative spectrum sensing (FD-SS) with half-duplex cooperative spectrum sensing (HD-CSS) and FD-CSS. Consistent with our

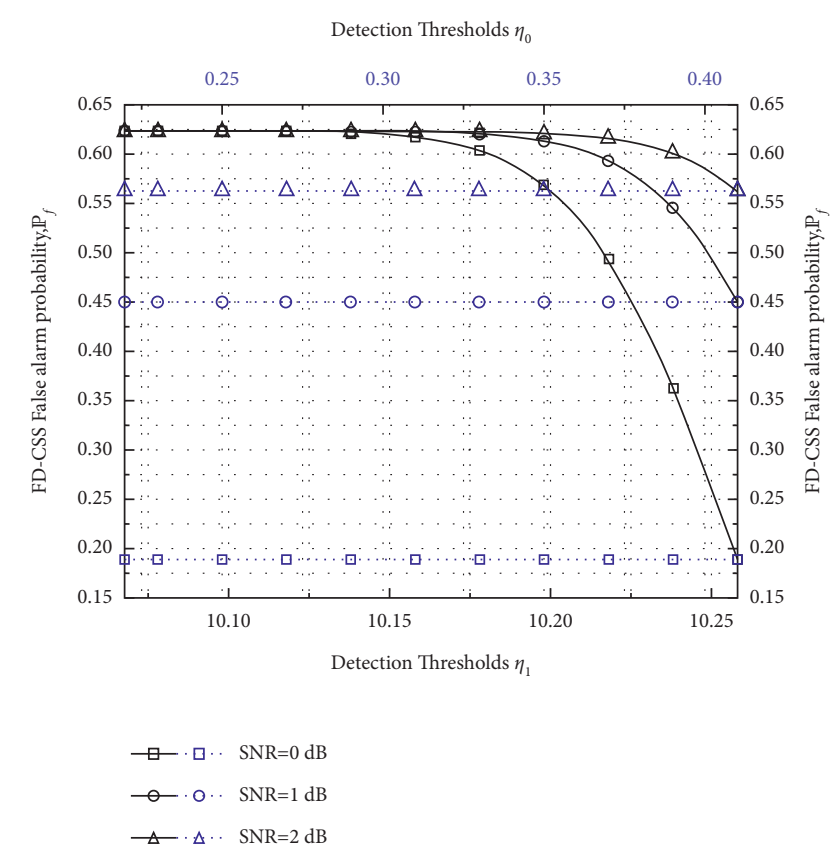

FIGURE 2: FD-CSS false alarm probability versus relative threshold. The lines and markers denote the results from the analysis and simulations, respectively.

previous theoretical analysis, under the limitation of detection probability, the throughput decreases with the decrease of the threshold. The simulation results show that FDCSS performs best when the throughout upper bound is not reached. The throughput of HD-CSS is a little higher than that of FD-CSS due to the fact that there is no selfinterference.

Figure 4 shows the change of throughput with SNR under different $n$ values. When SNR is small enough (in this scenario, less than $4 \mathrm{~dB}$ ), the throughput increases with the increase of $n$ as we analyzed in (40). It is mainly due to the fact that the false alarm probability decreases with the increase of $n$. When SNR is large enough (in this scenario, greater than $4 \mathrm{~dB}$ ), there is no difference in throughput with the increase of $n$. As we analyzed in (39), at this time, the upper limit of FD-CSS throughput is reached.

Figure 5 shows the transmission power-throughput curve of FD-CSS in terms of different RSI factor $\chi^{2}$ and SNR of the PU signal. When the transmission power is low, the secondary network throughput is better than HD-CSS [17]. The lines and markers denote the results from the analysis 


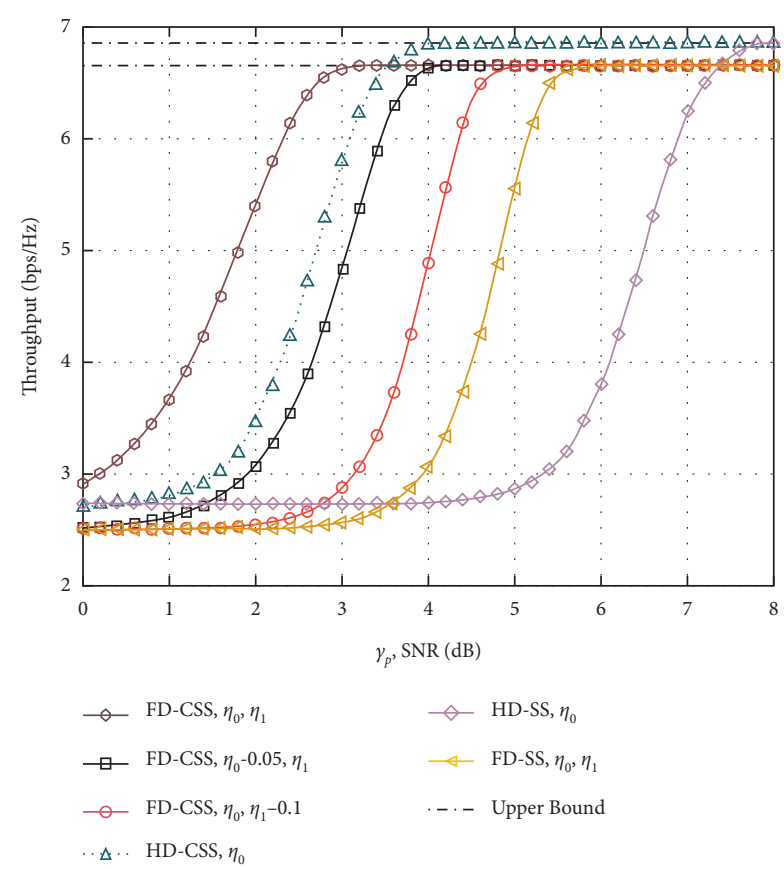

FIgURE 3: Throughput under different SNR.

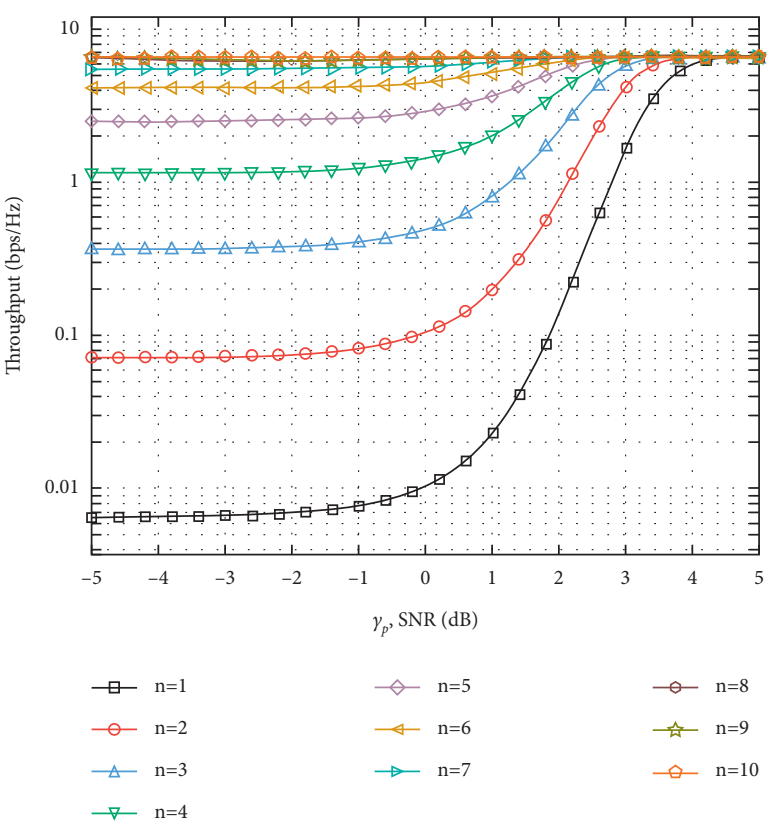

FIgURE 4: FD-CSS throughput versus relative the value of $n$ in the $n$-out-of- $N$ rule.

and simulations, respectively, and the asterisks $(O)$ denote the local optimal transmit power obtained by using Newton method. As we analyzed in equation (39), when the transmission power of FD-CSS increases, there is an extreme point in the power-throughput curve. The main reason is the existence of self-interference; when the transmission power increases, the self-interference will also increase leading to the increase of false alarm probability. When the RSI factor $\chi^{2}$ increases, the RSI increases, and the FD-CRN is easier to reach the extreme point.

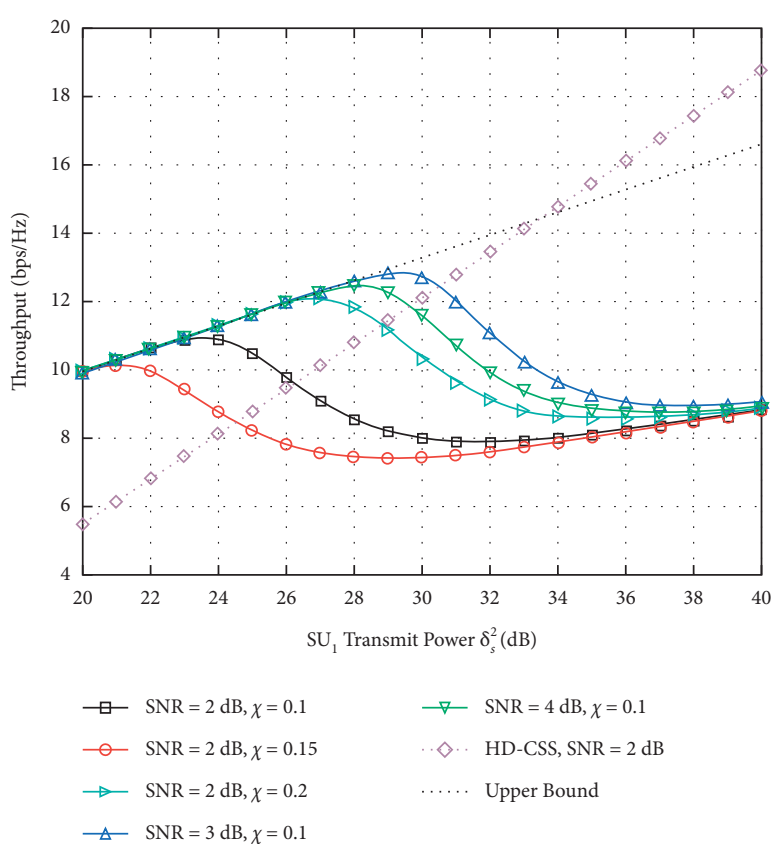

FIgURE 5: FD-CSS throughput versus relative transmission power.

\section{Conclusion}

In this paper, we considered the case of an FD-CRN, in which SUs are equipped with two antennas and have SIS capability and are thus able to perform simultaneous spectrum sensing and data transmission. Considering the limitation of detection probability in practical applications, we investigate how to set detection threshold to maximize secondary throughput under detection probability limitation. In CSS, SUs send their sensing results to $\mathrm{FC}$, and FC uses $n$ - out - of $-N$ decision rule to make decisions. We investigate how to set $n$ to maximize secondary throughput. From the perspective of energy efficiency, the local optimum of the transmission powerthroughput curve has the highest energy efficiency. We propose using Newton algorithm to obtain this local optimum. The simulation results show that the theoretical analysis is correct.

\section{Data Availability}

This study mainly focuses on theoretical analysis; the data are mainly Monte Carlo simulation verification. If readers want to get the data of the paper, contact the corresponding author via e-mail.

\section{Conflicts of Interest}

The authors declare that they have no conflicts of interest.

\section{Acknowledgments}

This work was supported by the National Natural Science Foundation of China (no. 62071481). 


\section{References}

[1] K. Shin, H. Kim, A. Min, and A. Kumar, "Cognitive radios for dynamic spectrum access: from concept to reality," IEEE Wireless Communications, vol. 17, no. 6, pp. 64-74, 2010.

[2] S. Sabat, P. K. Sharma, and A. Gandhi, "Full-duplex mobile cognitive radio network under Nakagami-m fading environment," AEU-International Journal of Electronics and Communications, vol. 17, pp. 64-74, 2019.

[3] X. C. Xia, K. Xu, Y. R. Wang, and Y. Y. Xu, “A 5G-enabling technology: benefits, feasibility, and limitations of in-band full-duplex mMIMO," IEEE Vehicular Technology Magazine, vol. 13, no. 3, pp. 81-90, 2014.

[4] K. Xu, Z. Shen, Y. Wang, X. Xia, and D. Zhang, "Hybrid timeswitching and power splitting SWIPT for full-duplex massive MIMO systems: a beam-domain approach," IEEE Transactions on Vehicular Technology, vol. 67, no. 8, pp. 7257-7274, 2018.

[5] F. Liu, X. Hou, and Y. Liu, "Capacity improvement for full duplex device-to-device communications underlaying cellular networks," IEEE Access, vol. 6, pp. 68373-68383, 2018.

[6] A. Nasser, A. Mansour, K. C. Yao, H. Abdallah, and H. Charara, "In-band full-duplex communication for cognitive radio," in Proceedings of the 23rd Asia-Pacific Conference on Communications, pp. 1-5, Perth, WA, Australia, December 2017.

[7] G. Liu, F. R. Yu, H. Ji, V. C. M. Leung, and X. Li, "In-band fullduplex relaying for $5 \mathrm{G}$ cellular networks with wireless virtualization," IEEE Network, vol. 29, no. 6, pp. 54-61, 2015.

[8] Z. Zhang, X. Chai, K. Long, A. V. Vasilakos, and L. Hanzo, "Full duplex techniques for 5G networks: self-interference cancellation, protocol design, and relay selection," IEEE Communications Magazine, vol. 53, no. 5, pp. 128-137, 2015.

[9] L. Zhang, M. Xiao, G. Wu, M. Alam, Y.-C. Liang, and S. Li, "A survey of advanced techniques for spectrum sharing in $5 \mathrm{G}$ networks," IEEE Wireless Communications, vol. 24, no. 5, pp. 44-51, 2017.

[10] D. Kim, H. Lee, and D. Hong, "A survey of in-band fullduplex transmission: from the perspective of PHY and MAC layers," IEEE Communications Surveys \& Tutorials, vol. 17, no. 4, pp. 2017-2046, 2015.

[11] G. Liu, F. R. Yu, H. Ji, V. C. M. Leung, and X. Li, "In-band fullduplex relaying: a survey, research issues and challenges," IEEE Communications Surveys \& Tutorials, vol. 17, no. 2, pp. 500-524, 2015.

[12] Z. Zhang, K. Long, A. V. Vasilakos, and L. Hanzo, "Fullduplex wireless communications: challenges, solutions, and future research directions," Proceedings of the IEEE, vol. 104, no. 7, pp. 1369-1409, 2016.

[13] J. Zhou, N. Reiskarimian, J. Diakonikolas et al., "Integrated full duplex radios," IEEE Communications Magazine, vol. 55, no. 4, pp. 142-151, 2017.

[14] Y. Liao, T. Wang, L. Song, and Z. Han, "Listen-and-Talk: protocol design and analysis for full-duplex cognitive radio networks," IEEE Transactions on Vehicular Technology, vol. 66, no. 1, pp. 656-667, 2016.

[15] I. F. Akyildiz, W.-Y. Lee, M. C. Vuran, and S. Mohanty, "NeXt generation/dynamic spectrum access/cognitive radio wireless networks: a survey," Computer Networks, vol. 50, no. 13, pp. 2127-2159, 2006.

[16] Y. Liao, T. Wang, L. Song, and Z. Han, "Listen-and-talk: fullduplex cognitive radio networks," in Proceedings of the IEEE Global Communications Conference, pp. 3068-3073, Austin, TX, USA, 2015.
[17] Y. Liao, T. Wang, L. Song, and B. Jiao, "Cooperative spectrum sensing for full-duplex cognitive radio networks," in Proceedings of the IEEE International Conference on Communication Systems, pp. 56-60, Macau, China, January 2015.

[18] P. V. Tuan and I. Koo, "Throughput maximisation by optimising detection thresholds in full-duplex cognitive radio networks," IET Communications, vol. 10, no. 11, pp. 1355-1364, 2016.

[19] H. Mokhtarzadeh, A. Taherpour, A. Taherpour, and S. Gazor, "Throughput maximization in energy limited full-duplex cognitive radio networks," IEEE Transactions on Communications, vol. 67, no. 8, pp. 5287-5296, 2019.

[20] C. Stevenson, G. Chouinard, L. Zhongding, H. Wendong, S. Shellhammer, and W. Caldwell, "IEEE 802.22: the first cognitive radio wireless regional area network standard," IEEE Communications Magazine, vol. 47, no. 1, pp. 130-138, 2009. 